\title{
The WATCH All-Sky Monitor for the Granat Project
}

Brandt, Søren; Lund, Niels; Rao, A. R.

Published in:

Advances in Space Research

Link to article, DOI:

10.1016/0273-1177(90)90148-S

Publication date:

1990

Document Version

Publisher's PDF, also known as Version of record

Link back to DTU Orbit

Citation (APA):

Brandt, S., Lund, N., \& Rao, A. R. (1990). The WATCH All-Sky Monitor for the Granat Project. Advances in Space Research, 10(2), 239-242. https://doi.org/10.1016/0273-1177(90)90148-S

\section{General rights}

Copyright and moral rights for the publications made accessible in the public portal are retained by the authors and/or other copyright owners and it is a condition of accessing publications that users recognise and abide by the legal requirements associated with these rights.

- Users may download and print one copy of any publication from the public portal for the purpose of private study or research.

- You may not further distribute the material or use it for any profit-making activity or commercial gain

- You may freely distribute the URL identifying the publication in the public portal

If you believe that this document breaches copyright please contact us providing details, and we will remove access to the work immediately and investigate your claim 


\title{
THE WATCH ALL-SKY MONITOR FOR THE GRANAT PROJECT
}

\author{
S. Brandt, N. Lund, A. R. Rao* \\ Danish Space Research Institute, Lundtoftevej 7, DK-2800 Lyngby, Denmark. \\ On leave from Tata Institute of Fundamental Research, Homi Bhabha Road, \\ Bombay - 400 005, Colaba, India.
}

\begin{abstract}
WATCH is an X-ray, all-sky monitor capable of localizing strong X-ray sources and following their time development. The GRANAT satellite, due to be launched in November 1989, carries several large $X$ - and gamma-ray telescopes and gamma-burst detector arrays.
\end{abstract}

Four WATCH units are included in the payload of GRANAT. We discuss the capabilities and potential scientific returns from this project.

Introduction Time variability is the outstanding characteristic of $\mathrm{X}$-ray astronomy. Although in some cases such variability has been found to exhibit periodic regularity, for many sources a large fraction of the observed change is irregular and, thus far, unpredictable.

The study of time variability of X-ray sources, therefore, remains an important branch of $\mathrm{X}$-ray astronomy. But the variability also introduces a new dimension in high-sensitivity spectroscopic studies of the sources. The proper interpretation of spectral data, or simply the ability to obtain spectral data at the right moment, may depend upon the knowledge of the activity of a given source over long periods of time.

Instruments, such as SIGMA, ART-P and ART-S on the GRANAT satellite (Roques, 1989), designed for high sensitivity spectral studies have, by nescessity, restricted fields-of-view and monitors only a few sources at any one time.

The WATCH instruments which have been designed specifically for time variability studies in a very wide field-of-view (4 steradians for each detector) will, however, complement the narrow-field instruments by providing the continous monitoring of the full X-ray sky. The highly eccentric orbit of the GRANAT satellite is also ideally suited for an all-sky monitor instrument.

Instrument principles WATCH is based on the rotation modulation collimator (RMC) principle (Mertz, 1968). The classical RMC is, however, not well suited for the study of X-ray sources (such as gamma-ray bursts) which vary on time scales comparable or shorter than the rotation period of the modulating grids.

WATCH uses only one modulating grid. The detector consists of two interleaved grids, each with the same pitch as the modulating grid. The separate signals from each of the two detector grids correspond exactly to the signal from a classical RMC but the two WATCH detector signals are $180^{\circ}$ out of phase. The sum of these two signals is not modulated by the rotating grid and can be used directly to monitor the fast time fluctuations of the source. The ratio of one of the signals to the sum signal is independent of the instantaneous intensity level of the source (as long as it is much brighter than anything else in the field) and, therefore, yields the instrument modulation function even for the rapidly flickering gamma-burst events (Lund, 1981).

Instrument characteristics The instrument field-of-view is characterized by a response function which drops to $50 \%$ at $57^{\circ}, 25 \%$ at $67^{\circ}$ and zero response at $73^{\circ}$ off axis angle. 
When the four WATCH units are mounted on the GRANAT satellite there is a significant overlap between the fields-of-view.

The rotation rate of the modulation assembly is 1 revolution per second which will allow the localization of even quite short gamma-ray bursts.

The interleaved detector grids form a "phoswich", an arrangement of alternating NaI(TI)- and CsI(Na)-scintillator strips, each $5 \mathrm{~mm}$ wide by $2 \mathrm{~mm}$ thick. The geometric area of the scintillator mosaic is $95 \mathrm{~cm}^{2}$. The energy range is from 6 to $180 \mathrm{keV}$ for Nal and from $15 \mathrm{keV}$ to $180 \mathrm{keV}$ for CsI. The lower energy threshold is set by absorbtion in the entrance window for $\mathrm{NaI}$ and by absorbtion in a deadlayer on the scintillator itself for CsI. The CsI deadlayer grows slowly with time - a fresh crystal may be sensitive down to $10 \mathrm{keV}$ and, after one year, the threshold will move up to about $15 \mathrm{keV}$.

The energy resolution of the detector is modest, about $30 \%$ FWHM at $60 \mathrm{keV}$. This is mainly due to the nonuniformity of the photocathode and the proximity of the scintillators and the phototube. The peak count rate capability of the WATCH detectors for the GRANAT mission is about $20,000 \mathrm{cps}$, which is about 50 times the expected count rate attributable to the diffuse $X$-ray background. The count rates, event timing and digitized pulse height data are collected into hardware registers and transferred from these registers to the instrument processor for every $2 \pi / 256$ angular increment of the modulation assembly - approximately every $4 \mathrm{msec}$. The arrival time of each event is determined relative to a clock running at $9600 \mathrm{~Hz}$ - the absolute time of this clock relative to UT will be known to an accuracy of $1 \mathrm{~ms}$.

Instrument data A processor collects and compresses the instrument data. The only data storage available for the WATCH instruments on GRANAT are the internal memories associated with the instrument processor. About $400 \mathrm{kbytes}$ are available for data storage in each instrument. (The four WATCH-units on GRANAT are completely independent of each other). The operations planning foresees telemetry sessions every 24 hours for three days and no contact on the fourth day of each orbit. Thus, we have available an average of about 37 bits/s for two days out of four and $18 \mathrm{bits} / \mathrm{s}$ for the other two days.

The instrument processor compresses the raw data stream from the detector into the following data types:

a) Modulation patterns. During quiet times RMC modulation patterns will be collected in two energy bands, 6-20 keV ( $N a I$ signals only) and 20-180 keV (NaI and CsI signals). The integration time for each pattern will be 512 s. Modulation patterns with better energy resolution ( 9 energy bands) will be collected simultaneously. The integration time will, in this case, be $4096 \mathrm{~s}$. These data will occupy about $60 \%$ of the available memory.

b) Count rates will be recorded continously with an integration time of $4 \mathrm{~s}$ in the two energy bands described above. (This requires about $15 \%$ of the available memory). The count rates of "hard X-rays" (above $180 \mathrm{keV}$ ) and "particles" (events yielding more than $2 \mathrm{MeV}$ ) will be recorded with an integration time of $128 \mathrm{~s}$.

c) Raw detector data. During gamma-ray bursts up to 100 kbytes of raw detector data may be stored. This corresponds to individual event timing and pulse height information for about $25,000 \mathrm{X}$-ray photons. (This requires $20 \%$ of the available memory).

Additional data types, with only small requirements on the memory resources are: energy calibration spectra, refined burst data with emphasis on source localization, energy spectra for selected, bright sources, period folded energy spectra for pulsating sources and instrument housekeeping data. Integration times for all the different data types may be modified by telecommand if the in-orbit experience with the instrument makes this desirable. 
Applications The applications can be divided into two groups: The study of time variations of known sources, and the detection and localization of new, transient sources.

The capabilities of the WATCH detectors on GRANAT for monitoring known sources have been evaluated by calculating the instrument performance for the sources in the HEAO-A4 catalog (Levine et al, 1984). It appears, that WATCH can monitor all the HEAO-A4 persistent sources for variablity on timescales ranging from a few seconds for Sco X-1 to about 3 weeks for Mkn 509. The ability to monitor simultaneously the activity level of close to a hundred known sources has been confirmed through extensive computer simulations.

By alerting the pointed instruments on GRANAT or observatories on the ground to unusual activity in flare stars, $X$-ray binaries or extragalactic sources important spectral data may be obtained during such hectic stages of the source development.

For transient sources a safe detection and localization requires the signal to exceed the noise by 10 sigma. About 10 new $X$-ray transients per year and 40 fast transients per year are expected according to the searches made in the ARIEL-V and HEAO-AI databases (Pye and McHardy, 1983 and Connors, 1988).

WATCH will continously monitor the entire sky for fluctuations in the background count rate which may indicate the onset of an intense $X$ - or gamma-ray burst. The trigger logic is set to respond to nonstatistical increases in the count rates on 14 different time scales between $4 \mathrm{~ms}$ and $32 \mathrm{~s}$. If a burst is detected, burst data processing will be triggered which will aim at the best possible recording of the modulation pattern, the time structure and the spectral evolution of the burst. Based on the statistics from the KONUS experiments on the VENERA 11 and 12 spacecraft (Mazets et al, 1981) we expect to detect about 100 gamma bursts per year and to be able to locate about two thirds of them to better than 10 arcminute accuracy. The opportunities for rapid (within 24 hours) follow-up observations from the ground of $\mathrm{X}$ - and gamma-burst error boxes opens intriguing perspectives for pinning down the elusive sources of these bursts.

Finally it must be mentioned that both the Sun and the Earth will be continously monitored for their X-ray emission and, in both cases, these data will be analyzed and correlated with other observations.

Conclusions The inclusion of four wide field $\mathrm{X}$-ray monitors into the GRANAT payload opens up new perspectives for the study of the time variable $X$-ray sky. For the first time it will be possible to perform a sensitive search for sources showing transient behaviour in the $5 \mathrm{~s}$ to $5000 \mathrm{~s}$ interval which have been difficult to access either with collimated instruments in low Earth orbit or omnidirectional instruments in interplanetary space. The continous record of activity for the 60 to 100 brightest $X$-ray sources in the sky will serve as an important reference for any specific observation of these sources in the coming years.

The full potential of the expected data from WATCH will, however, only be realized if the detection of transient phenomena is followed up with the minimum possible delay by other instruments with greater sensitivity (GRANAT, GINGA, GRO, ROSAT, ST etc.) and from the ground, in the optical, infrared and radio bands. For this reason it is our intention to reduce the data on the ground very fast (on a day-to-day basis) and to set up a rapid data dissemination network. Anyone interested to take part in such follow-up investigations is invited to contact the authors.

Acknowledgements The development of WATCH has been supported by the Danish Space Board and the Natural Sciences Research Council. The authors also want to acknowledge the efforts by our colleagues at the Space Research Institute of the Soviet Academy of Sciences which mad it possible to accomodate the four WATHC units on GRANAT in less than two 
years, and also provided a very usefull calibration facility for the energy calibration of the WATCH instruments.

\section{References}

P. Roques, The SIGMA telescope for the GRANAT mission, Space Research, 1989.

A. Mertz, in Proc. Mod. Optics, Polytech. Inst. of Brooklyn (1968)

N. Lund, Ap. Sp. Sc., 75, p. 145 (1981).

A. M. Levine et al, Ap. J. Suppl. Ser. 54, p. 581-617 (1984).

J. P. Pye and I. M. McHardy, Mon. Not. R. Astr. Soc., 205, p. 875-888 (1983).

A. Connors, NASA Goddard Laboratory for High Energy Astrophysics 88-014 (1988).

E. P. Mazets et al, Ap. Sp. Sc., 80, 1, p. 3 (1981).
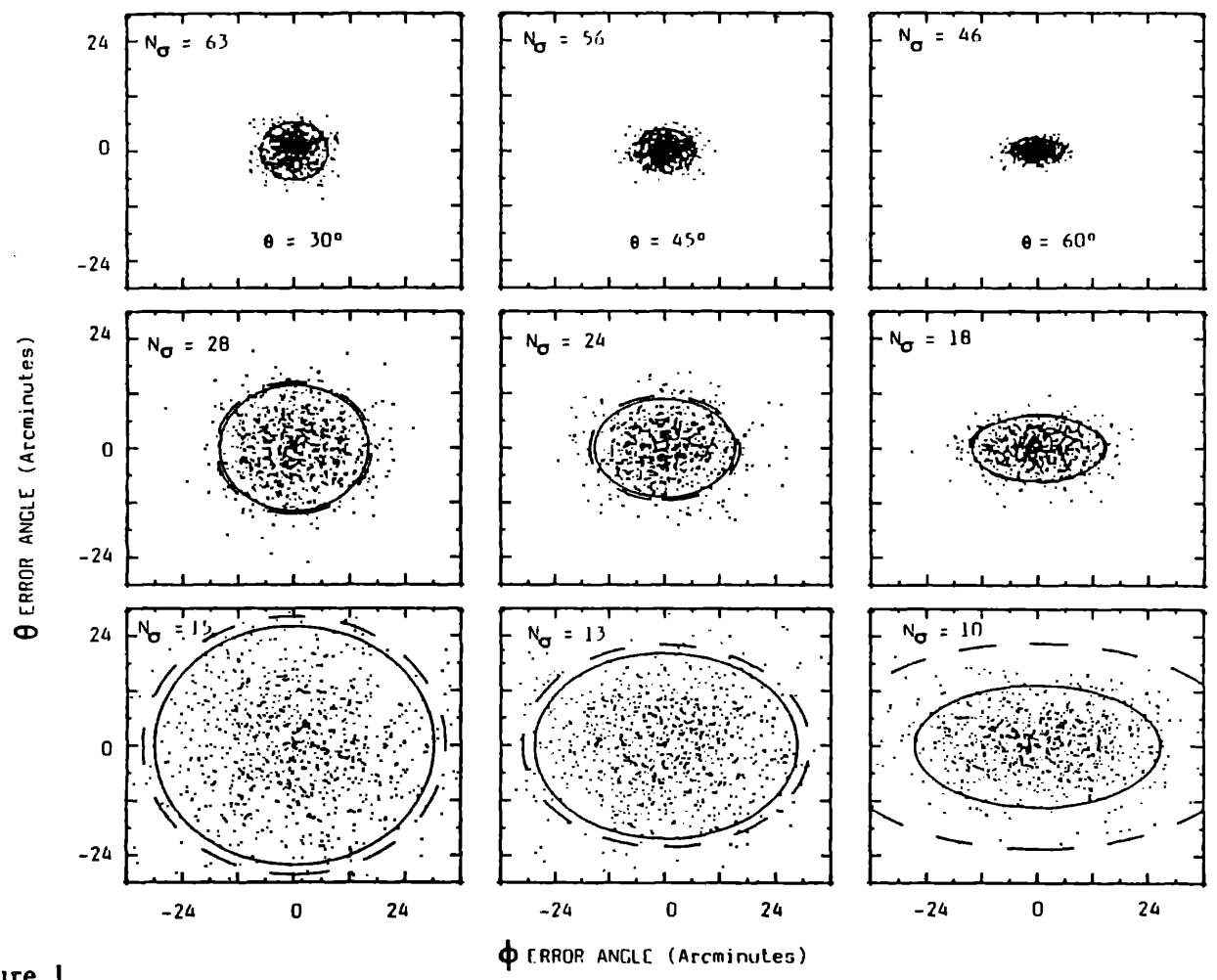

Figure 1.

Q TRROR ANGLL (Arcminutes)

Monte Carlo simulations of the localization capabilities of WATCH. The simulation parameters were: Top: 2 second burst with mean signal = $12 \times$ background, Middle: 10 second burst with mean signal = background, Bottom: 2 second burst with mean signal $=1.2 \mathrm{x}$ background.

The theoretical $1 \sigma$ error radius is given by:

$$
r_{1 \sigma} \leq 2.4^{\circ} \quad N_{\sigma}^{-1 / 2} \text { with } N_{\sigma}=\frac{\text { Signal (photons) }}{\sqrt{\text { Signal + Background (photons) }}}
$$

As shown in the figure the localization improves with increasing off-axis angle, $\theta$, despite the $\cos \theta$ reduction in the number of detected photons.

The full curves showns the theoretical $90 \%$ confidence contours, the dashed curves are based on the Monte Carlo results. For the weakest bursts shown, the localization is lost for a significant fraction of the cases for large values of the off-axis angle. However, the these angles the bursts will be observed by more than one WATCH instrument on GRANAT. 